\title{
Brain maturation and damage in infants dying from chronic pulmonary insufficiency in the postneonatal period
}

\author{
J. F. SMITH, E. O. R. REYNOLDS, and A. TAGHIZADEH \\ From the Departments of Morbid Anatomy and Paediatrics, University College Hospital and Medical School, \\ London
}

\begin{abstract}
Smith, J. F., Reynolds, E. O. R., and Taghizadeh, A. (1974). Archives of Disease in Childhood, 49, 359. Brain maturation and damage in infants dying from chronic pulmonary insufficiency in the postneonatal period. The changes in the brains of 11 infants who survived from 29 days to 13 months after severe chronic pulmonary insufficiency are described.

Brain maturation as assessed by myelination and gyral formation was within normal limits. In 5 infants the brain weight was less than normal if age was used in the comparison, but greater than normal if body weight was used.

In addition to hypoxic or ischaemic damage, which was found in some degree in all cases except one, there were several other local lesions. These included periventricular leucomalacia 7 , periventricular haemorrhage 1 , hydrocephalus 2 , and cystic encephalomalacia 3. Though the last is almost certainly due to vascular occlusion, this could not be shown. The possibility of cerebral embolism after umbilical vein catheterization is considered but not proven.
\end{abstract}

In the last decade it has become possible with modern therapy to keep alive many babies of low birthweight who would previously have died. Most of these survive and develop normally without neurological or mental defect despite such hazards as hyaline membrane disease and apnoea of prematurity (Rawlings et al., 1971; Stewart, 1972). However, some die within the first few days of life with a combination of lung and brain lesions. Among the latter, intraventricular and other cerebral haemorrhage consequent to rupture of subependymal plate haemorrhage are perhaps the most florid, but hypoxic or ischaemic damage in the hemispheres and brain stem is also important (C. Rodeck and J. F. Smith, in preparation). In the present paper we describe changes in the brain in 11 infants with severe lung disease causing hypoxia, who survived for 29 days to 13 months. This survival period was chosen because it was thought that 4 weeks was the minimum time in which it would be possible to assess whether the period of stress or any consequent local lesion had affected brain maturation. A further infant who died at 27 days from septicaemia without previous hypoxia is included as a control.

Received 1 October 1973.

\section{Material and methods}

All the infants were treated in the Neonatal Unit at University College Hospital, 4 were born at the hospital and 8 , including the majority of those with severe brain damage, were born elsewhere and transferred to this hospital because of low birthweight or respiratory illness.

Clinical features. The 11 infants with chronic pulmonary insufficiency were born at 24 to 36 weeks' gestation (mean 29 weeks), weighing 750 to $2000 \mathrm{~g}$ (mean $1347 \mathrm{~g}$ ). None of them weighed less than the 10th centile for gestation (Lubchenco et al., 1963). No significant complications of pregnancy or delivery were present in 7 infants: in the remaining 4 severe preeclamptic toxaemia, severe rhesus isoimmunization, concealed accidental haemorrhage, and abruptio placentae with consequent cardiac arrest at delivery each occurred once. 4 infants breathed immediately at birth, but the remainder needed endotracheal intubation. In addition to the infant with cardiac arrest, 3 others were noted as having suffered severe birth asphyxia with subsequent base excess (BE) values of -12 to -25 $\mathrm{mEq} / \mathrm{l}$. The reason for mechanical ventilation was hyaline membrane disease in 4 infants and apnoea due to immaturity in the remainder. The duration of ventilation ranged from 1 to 12 weeks (mean 5 weeks) and all the infants developed clinical evidence of severe lung damage. Hypoxic episodes occurred during or after ventilation in 9 of the 10 infants, as judged by an arterial 
oxygen tension below $30 \mathrm{mmHg}$ ( 2 infants) or a sudden fall in $\mathrm{BE}$ of $8 \mathrm{mEq} / \mathrm{l}$. or more after an episode of cyanosis and bradycardia. Blood glucose levels were repeatedly measured but only one value below $25 \mathrm{mg} / 100 \mathrm{ml}$ was recorded. All the infants displayed abnormal neurological signs; 3 had fits, 2 appeared decerebrate after severe hypoxic episodes on the last day of life, and all at some time showed abnormalities of posture, tone, responsiveness, or development.

The control infant weighed $3120 \mathrm{~g}$ at 37 weeks' gestation. He was operated upon for multiple intestinal atresias but finally succumbed to klebsiella septicaemia on the 27th day. He never showed any evidence of hypoxia or neurological abnormality.

Necropsy. Most of the necropsies were done by one of us (A.T.), and the bulk of the observations were made on brains fixed by immersion in formalin and examination of paraffin and frozen sections taken a few weeks later. Luxol fast blue stain was used for the demonstration of myelin, and Holzer and phosphotungstic acidhaematoxylin preparations for glia cells and gliosis, supplemented by metallic impregnation methods in some cases. In the assessment of myelination, the data of Yakovlev and Lecours (1967) were used and those of Larroche (1962) for correlation of gyral maturation and age. There are no adequate data for assessing the normality of brain weight with age or body weight in a series such as this where premature birth was followed by a survival period of weeks or months. The tables of Gruenwald (1963), in which brain weight is compared with gestational age and body weight in newborn infants have therefore been used in assessing the significance of the brain weight in all cases except 11 where the data of Schulz, Giordano, and Schulz (1962) were appropriate. The age of the cases was taken as the sum of the gestational age and neonatal survival period. This varied from 28 to 44 weeks except for Case 11 .

\section{Results}

The essential pathological observations are recorded in Tables $I$ and II. Table I shows that maturation as judged by gyral formation and myelination had continued normally despite the severity of the stress and the presence of local lesions in the majority, which did have an effect on local development. In 5 of the infants, (Cases 1, 2, 5, 6, and 9) the brain weight was less than would have been expected for a 'normal' infant of an age equal in weeks to the sum of the gestational age and the neonatal survival period. In all of these, however, the brain weight was greater than would have been expected from the body weight of the infant if the neonatal figures of Gruenwald are used for comparison.

Table II summarizes the local and general lesions present. All except Case 11 had focal areas of damage in grey or white matter, which are described as hypoxic-ischaemic (HID) as it is not always possible to decide whether the tissue hypoxia is a result of a lowered $\mathrm{Po}_{2}$ or diminished perfusion. The extent and degree of the lesions varied considerably, being more severe in Cases 5 and 7 than in the others, but in no instance was there infarction of the grey matter. In many the presence of karyorrhexes in grey matter of hemispheres or brain stem (Fig. 1) suggested that hypoxic damage occurred in the terminal days of life as well as at birth or soon after. A few foci of grey matter damage were present in the hemispheres in the control, Case 12. These were, however, much less prominent than in Cases 1 to 10 .

The more severe local lesions given in Table II are subependymal plate haemorrhage (SPH), periventricular leucomalacia (PVL), periventricular haemorrhage $(\mathrm{PVH})$, and cystic encephalomalacia (CE). A lesion that was mainly haemorrhagic was found once and hydrocephalus as a result of subependymal plate haemorrhage twice. The latter, which is one of the most serious, if not the

TABLE I

Relation of gyral development and myelination (expressed in terms of 'fetal age' except for Case 11) to age (sum of gestation + age after delivery) and relation of brain weight to age and body weight

\begin{tabular}{|c|c|c|c|c|c|c|c|}
\hline Case & Sex & $\begin{array}{l}\text { Gestation } \\
\text { (wk) }\end{array}$ & $\begin{array}{l}\text { Age at death } \\
(\mathbf{w k})\end{array}$ & $\begin{array}{l}\text { Body weight at } \\
\text { necropsy (g) }\end{array}$ & $\begin{array}{c}\text { Brain weight } \\
\text { (g) }\end{array}$ & $\begin{array}{l}\text { Gyral age } \\
\text { (wk) }\end{array}$ & $\begin{array}{l}\text { Myelination age } \\
(w k)\end{array}$ \\
\hline $\begin{array}{r}1 \\
2 \\
3 \\
4 \\
5 \\
6 \\
7 \\
8 \\
9 \text { twin } \\
10 \\
11 \\
12\end{array}$ & $\begin{array}{l}\mathbf{F} \\
\mathbf{M} \\
\mathbf{M} \\
\mathbf{M} \\
\mathbf{F} \\
\mathbf{M} \\
\mathbf{M} \\
\mathbf{M} \\
\mathbf{F} \\
\mathbf{M} \\
\mathbf{M} \\
\mathbf{M}\end{array}$ & $\begin{array}{l}24 \\
24 \\
31 \\
24 \\
27 \\
30 \\
28 \\
33 \\
27 \\
32 \\
36 \\
37\end{array}$ & $\begin{array}{c}4 \\
12 \\
13 \\
13 \\
9 \\
5 \frac{1}{2} \\
8 \frac{1}{2} \\
6 \\
4 \\
6 \frac{1}{2} \\
56 \\
4\end{array}$ & $\begin{array}{r}670 \\
1220 \\
3440 \\
2140 \\
1570 \\
1720 \\
1765 \\
2170 \\
1300 \\
2290 \\
6180 \\
3220\end{array}$ & $\begin{array}{l}150 \\
230 \\
410 \\
330 \\
270 \\
240 \\
290 \\
320 \\
245 \\
340 \\
980 \\
430\end{array}$ & $\begin{array}{c}26 \\
40 \\
40 \\
40 \\
36 \\
36 \\
36 \\
40 \\
32 \\
40 \\
1 \mathbf{y r} \\
40\end{array}$ & $\begin{array}{c}26 \\
38 \\
40 \\
40 \\
36 \\
36 \\
34 \\
36 \\
32 \\
38 \\
1 \mathbf{y r} \\
40\end{array}$ \\
\hline
\end{tabular}


TABLE II

Local lesions present in brains

\begin{tabular}{cl}
\hline Case no. & \multicolumn{1}{c}{ Local lesions } \\
\cline { 2 - 2 } 1 & HID, PVL, slight SPH \\
2 & HID, CE with haemorrhage \\
3 & HID, PVL \\
5 & HID, CE with some old haemorrhage \\
6 & HID, CE \\
7 & HID, PVL; old meningeal and \\
8 & cerebellar haemorrhage \\
9 & HID, PVL \\
10 & HID, PVL, SPH, hydrocephalus \\
11 & HID, PVL, SPH, hydrocephalus \\
12 (control) & HID, PVL, PVH; cerebellar \\
& hil \\
& Minimal HID \\
\end{tabular}

HID, hypoxic ischaemic damage; PVL, periventricular leucomalacia; SPH, subependymal plate haemorrhage; $C E$, cystic encephalomalacia; PVH, periventricular haemorrhage.

most serious hazard of prematurity at the present time, does not figure greatly in this series because if intraventricular extension occurs it usually causes death in the first week of life. However, a slighter intraventricular bleed may lead to hydrocephalus, as in Cases 8 and 9. This is attributed to organization of the haemorrhage in the meninges or at the exits of the fourth ventricle with obstruction to CSF flow (Smith, 1974). In Case 1 this complication did not develop, though evidence of old haemorrhage was present in the subependymal plate and the meninges.

In periventricular leucomalacia (Banker and Larroche, 1962), foci of coagulation necrosis in the future white matter of the hemispheres are followed by glial scars (Fig. 2). Our Cases 1, 3, 6, 7, 8, and 9 had such scars, though not always in the distribution described by Banker and Larroche-the tissue adjacent to the frontal horns, the superolateral angles of the lateral ventricles, and superior to the occipital horns. On the whole they were not so severe as those described by these authors and in only one was the initial coagulation necrosis still evident.

We have used the term 'old periventricular haemorrhage' to describe the lesions in Case 10. This indicates a lesion similar to periventricular leucomalacia in distribution but one in which evidence of old haemorrhage was dominant (Fig. 3 and 4). This is indeed the only case in our experience in which the tissue damage was similar to that described by Schwartz (1961) and attributed by him to stasis in the Galenic venous system.

Cystic encephalomalacia-areas in which disintegration had occurred (Fig. 5) with cavities surrounded by gliosis-were present in Cases 2, 4, and 5 , accompanied by evidence of severe local haemorrhage in 2 and slighter haemorrhage in 5. The areas involved were the left temporal lobe in 2 , the left temporal, left frontal, and both parietal lobes in 4 , and the left lateral temporal and occipital lobes in 5. The damage involved subcortical tissue to a greater extent than cortex, but local thrombosis was not detected.

Evidence of old haemorrhage not attributable to an extension from subependymal plate haemorrhage was present in the leptomeninges in Case 6 and in

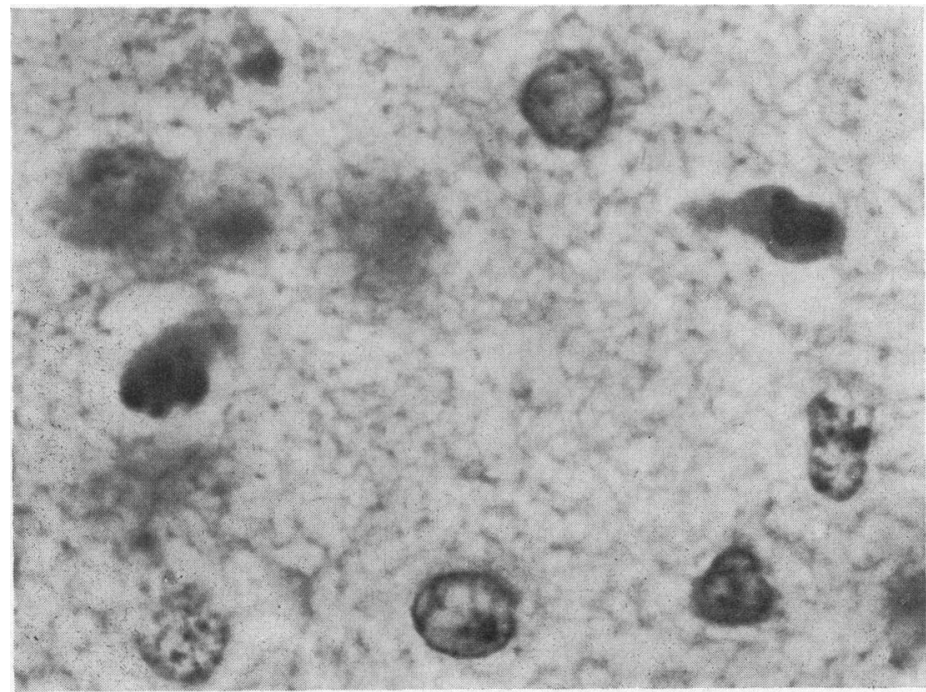

FIG. 1.-Case 6. Karyorrhexes of neurones in the ventral pons. (H. and E. $\times 1000$.) 


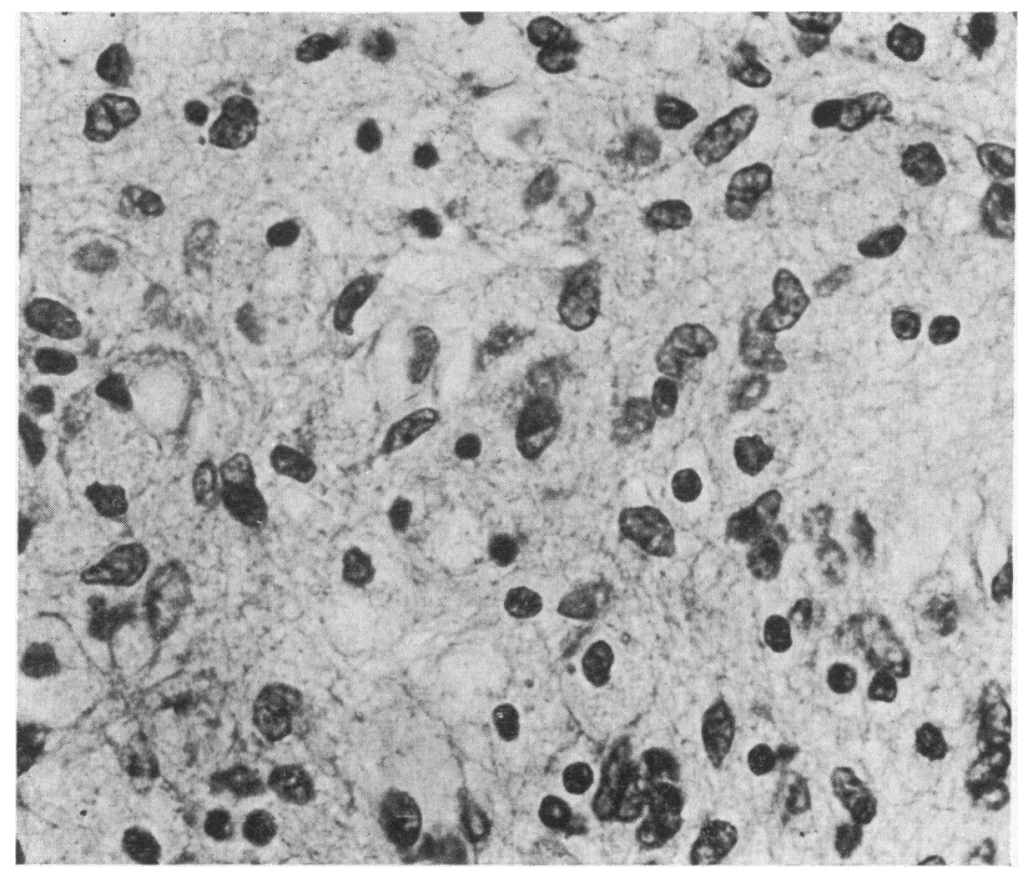

Fig. 2.-Case 5. Mixed glia proliferation in white matter-a late stage of periventricular leucomalacia. (H. and $E$. $\times 355$.)

isolated cerebellar folia in Cases 6 and 10. The subependymal pseudocyst present in Case 10 (Fig. 6) showed residual groups of primitive neuroblasts adjacent to it, small microcysts, and some gliosis in the adjacent residual matrix, but no old haemorrhage in this site.
An increase in vascularization of some brain stem nuclei was present in Cases 1 and 4 similar to that described by Brand and Bignami (1969). Subependymal gliosis in the lateral part of the occipital horn was present in all cases and was seen in other parts of the ventricular system in a few, this being a

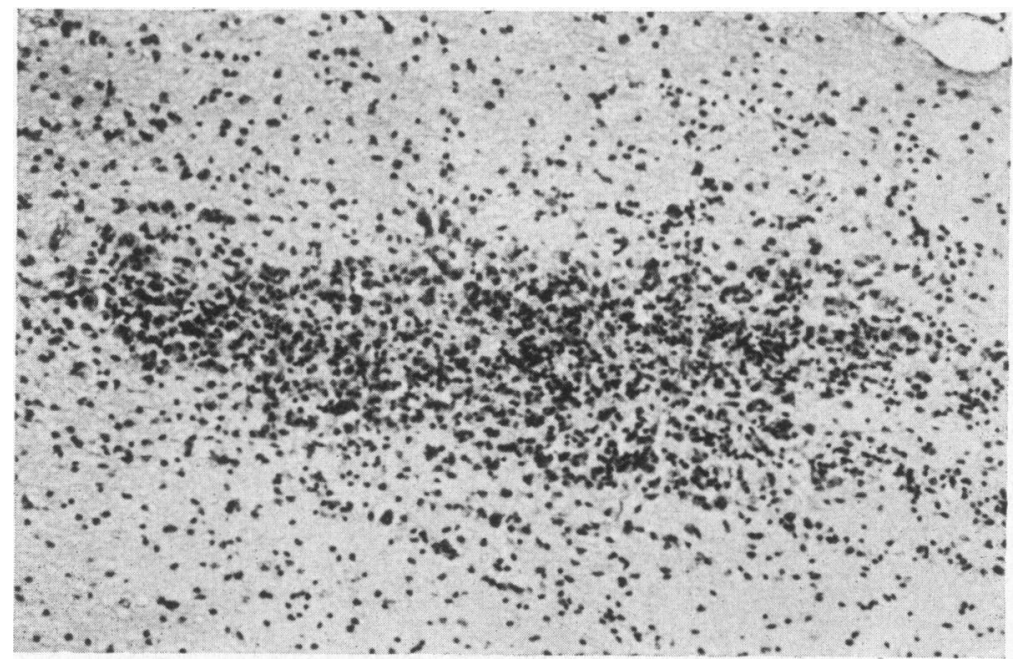

FIG. 3.-Case 10. Dense microglia reaction at site of old periventricular haemorrhage. (H. and E. $\times 100$. 


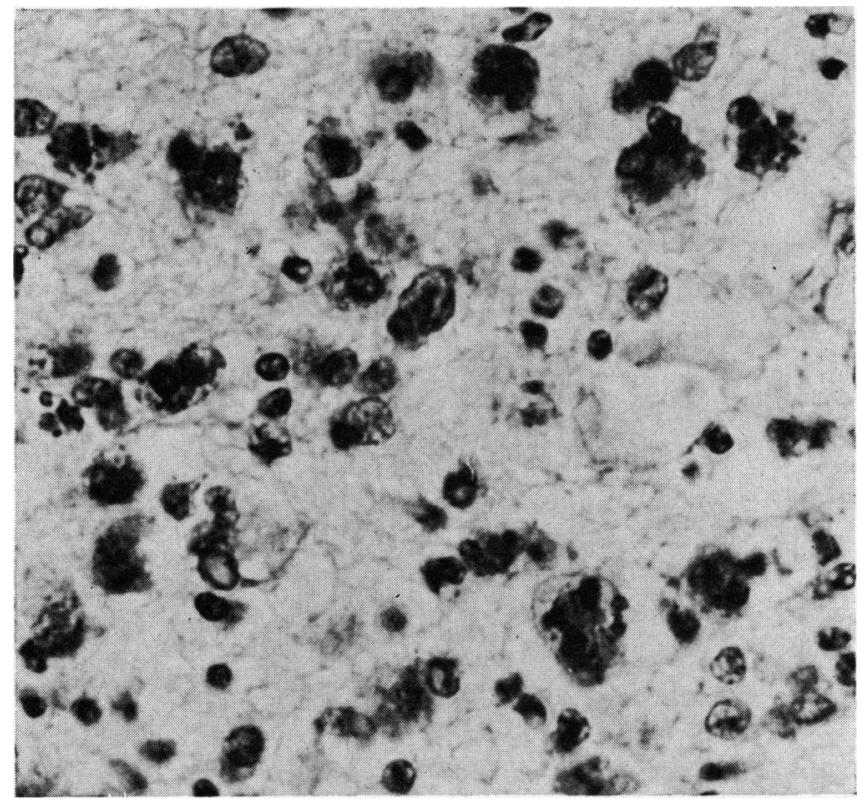

FIG. 4.-Case 10. Blood pigment and lipid in microglia cells at site of old periventricular haemorrhage. $\quad(P A S . \quad \times 400$.

lesion which is common in neonatal brains and not diagnostic of any particular pathological process. The widespread fibrillary gliosis also described by Brand and Bignami (1969) was not so frequent or extensive in our cases, though areas were seen in the

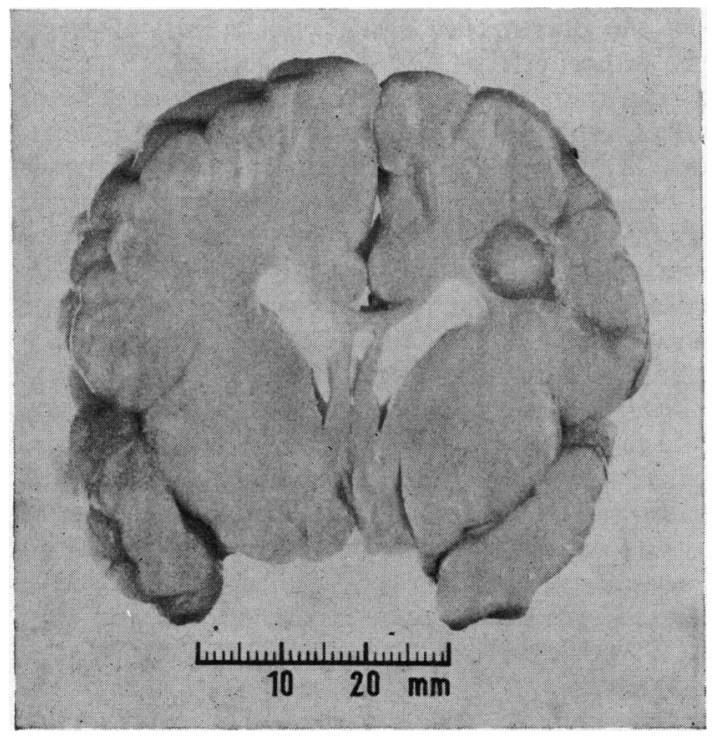

Fig. 5.-Case 4. Cystic encephalomalacia in left frontal lobe. medullary olives in Cases 3, 4, and 6 (Fig. 7) and in the thalamus and hemisphere white matter in Case 11.

\section{Discussion}

This study was initiated when it became obvious that a few infants (Cases 2, 4, and 5) who survived for some months with severe lung damage developing after prolonged mechanical ventilation had unusual local lesions in the brain, though maturation had not been affected as judged by gyral formation and myelination. The whole series indicates that gyral formation and myelination continue normally despite severe neonatal stress and episodes of hypoxia. No evidence of hypermyelination was seen, but the survival period was perhaps too short for this to develop.

As mentioned in Results, some of the infants had brains below normal weight for their age if the latter is calculated as the sum of the gestational age +age after delivery. Such a low brain weight, however, was associated with a low body weight for such an 'age'. When the comparison of brain weight and body weight rather than age is used, the brain weights in these infants appear above normal. It may be recalled that Gruenwald (1963) recorded high brain weights in infants with chronic fetal distress-another condition in which brain growth is not retarded in the same way as body growth. There is therefore no evidence from this material 


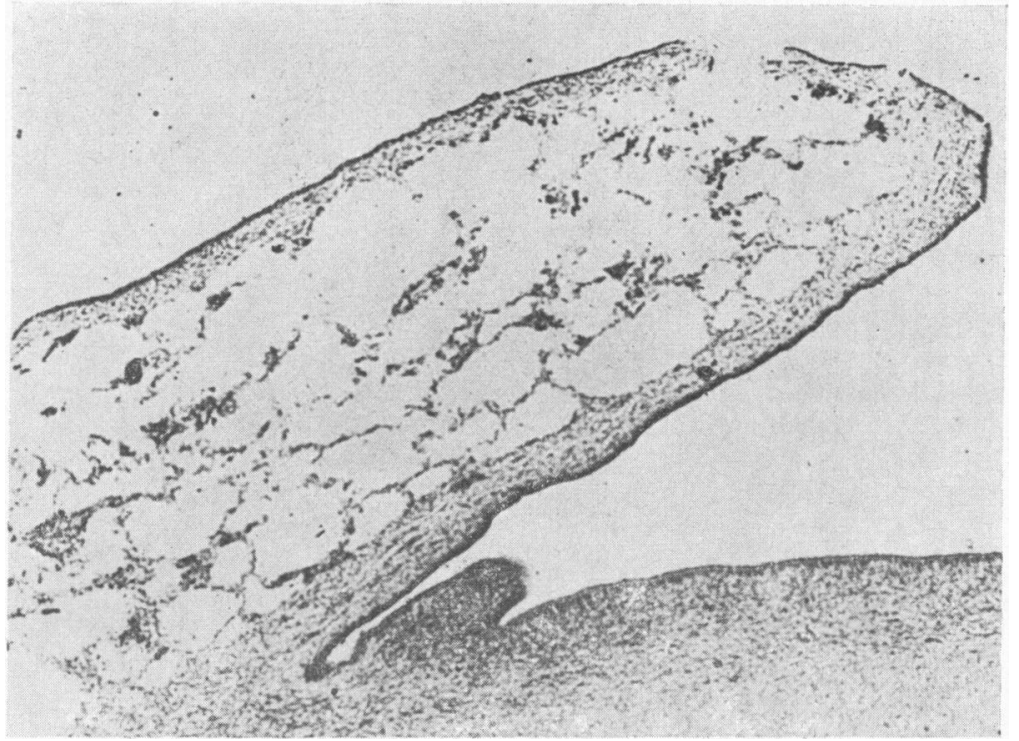

Fig. 6.-Case 10. Subependymal pseudocyst. Residual neuroblasts between cavities. (H. and E. $\times 34$.

that chronic pulmonary insufficiency in the newborn leads to defective growth or anatomical maturation of the brain.

The other questions to be considered are the pathogenesis and effects of the local lesions. Minor degrees of hypoxic or ischaemic damage are present in the majority of perinatal deaths and were present in the one control case in this series, i.e. a death without pulmonary insufficiency. Such lesions were more extensive in all the remaining infants

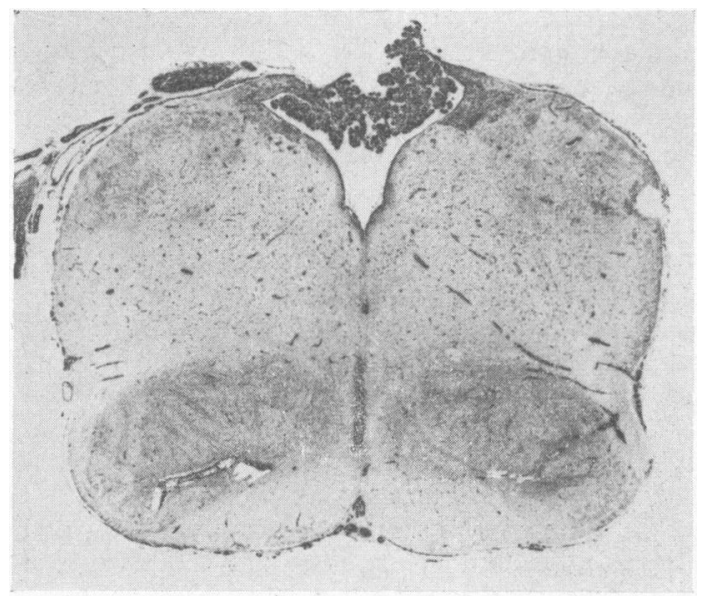

FIG. 7.-Case 6. Fibrillary gliosis of the medullary olives. $($ Holzer. $\times 6$.) except the one (Case 11) in which death occurred at 13 months and in whom none were detected. This infant had definite clinical evidence of developmental retardation for which the cause is not clear. Possibly cerebral damage had occurred without leaving detectable scars, or the retardation might have been attributable to the severe general illness and confinement.

At the present time we cannot assess the longterm clinical effects of moderate degrees of hypoxic or ischaemic damage or of the moderate degrees of periventricular leucomalacia seen in several infants. Both these lesions are probably due to hypoxic episodes which were recorded at various times in all the infants. In no case was there extensive devastation of nervous tissue of the type which sometimes results from severe hypoxia and/or ischaemia in the perinatal period; the most severe lesions were in Cases 6 and 7.

The cystic encephalomalacia lesions seen in Cases 2,4 , and 5 are most like those classified as focal cortical and subcortical encephalomalacia by Wolf and Cowen (1954) and attributed by them to anoxia, possibly caused by vascular occlusion or infection. In their review of 17 cases they were able to find reported evidence of such vascular occlusion in only 4. We could not show vascular occlusion, but the focal nature of the lesions makes it difficult to attribute them to anything else-they are virtually areas of infarction. The reaction in the surrounding tissue was comparable to that found 
with such lesions in later infancy or adult life except for the fewer lipid macrophages-possibly because of the absence of myelination in the sites at the time of the lesions. The reaction indicated a lesion of some weeks' duration and probably occurring in early neonatal life.

While the pathogenesis of these lesions remains unclear, it is interesting that all 3 infants displaying them had had umbilical venous catheters in position for between 3 and 7 days. In one infant the catheter had been replaced because of a blockage at its tip. It is possible that thrombi from these catheters passed through the foramen ovale, which often shunts blood from the right to the left atrium in hypoxic infants (Stahlman, 1966) and thence into cerebral arteries, causing infarction. However, we have no proof for this suggestion and 3 of the remaining 9 infants also had umbilical vein catheters inserted without developing similar lesions.

The detailed description of the hydrocephalic brains is given elsewhere (Smith, 1974). This complication in survivors of subependymal plate haemorrhage is not invariable, as Case 1 showed old haemorrhage in the ependymal plate and the meninges without hydrocephalus: it is possible that it would have developed if this infant had survived longer. Small haemorrhages in isolated cerebellar folia in Cases 6 and 10 have no obvious pathogenesis.

The evidence of old haemorrhage in the periventricular sites in Case 10 is more rationally explained as a sequel to neonatal asphyxial heart failure with increased venous pressure and rupture of veins as suggested by Cole et al. (1974) in the pathogenesis of subependymal plate haemorrhage than as a consequence of Galenic vein kinking during delivery. It was associated with pseudocystic change but not haemorrhage in the subependymal plate. Similar pseudocysts have been described by Larroche (1972) who found evidence of old haemorrhage in only 4 of 22 cases. She reviewed the associated factors in mothers and infants and suggested that the very cellular and actively growing subependymal plate might be susceptible to a variety of injuries such as infections, blood incompatibility, and even that causing associated malformations. Case 10 had rhesus isoimmunization as well as bronchopulmonary dysplasia, but we have not seen such pseudocysts in association with either of these conditions previously. The lung pathology was, however, sufficiently severe to cause cor pulmonale and it is possible that the heart failure led to damage in the active germinal matrix a little later than the first few days of life and at a time when the veins were less liable to rupture.

An attempt can be made to assess the long-term effects of some of these macroscopical lesions. The areas of cystic encephalomalacia would be expected to interfere with function in strategic areas, but the well-known capacity of the growing brain to adapt would have possibly mitigated this, except in Case 4 where the lesions were extensive. The multiple sites of old haemorrhage in Case 10 would again have been expected to leave some residual defect, but the effects of the pseudocyst in the ependymal plate are not known. One occasionally sees a small cystic area in this site in necropsies on adults without a history of long-term neurological disease. The effects of the hydrocephalus such as that in Cases 8 and 9 would be determined by the residual damage in the plate, the efficiency of shunt therapy, and other lesions. In Case 8 the residual damage in the plate and the extent of hypoxia or ischaemia was considerable and the shunt had not been effective. In Case 9 there was much less damage in the plate, but significant hypoxic damage; shunt therapy was not undertaken because of the severity of the lung condition which was complicated by an episode of massive pulmonary haemorrhage at 3 weeks of age.

This work was assisted by grants from the National Fund for Research into Crippling Diseases and University College Hospital Research Funds.

\section{REFERENCES}

Banker, B. Q., and Larroche, J. C. (1962). Periventricular leukomalacia of infancy. Archives of Neurology, 7, 386.

Brand, M. M., and Bignami, A. (1969). The effects of chronic hypoxia on the neonatal and infantile brain. Brain, 92, 233.

Cole, V. A., Durbin, G. M., Olafson, A., Reynolds, E. O. R., Rivers, R. P. A., and Smith, J. F. (1974). Pathogenesis of intraventricular haemorrhage in pre-term infants. Submitted for publication.

Gruenwald, P. (1963). Chronic fetal distress and placental insufficiency. Biology of the Neonate, 5, 215.

Larroche, J. C. (1962). Quelques aspects anatomiques du développement cérébral. Biology of the Neonate, 4, 126.

Larroche, J. C. (1972). Subependymal pseudo-cysts in the newborn. Biology of the Neonate, 21, 170.

Lubchenko, L. O., Hansman, C., Dressler, M., and Boyd, E. (1963). Intrauterine growth as estimated from liveborn birthweight data at 24-42 weeks of gestation. Pediatrics, 32, 793.

Rawlings, G., Reynolds, E. O. R., Stewart, A. L., and Strang, L. B. (1971). Changing prognosis for infants of very low birth weight. Lancet, 1, 516.

Schulz, D. M., Giordano, D. A., and Schulz, D. H. (1962). Weights of organs of fetuses and infants. Archives of Pathology, 74, 244.

Schwartz, P. (1961). Birth Injuries of the Newborn. Hafner, New York.

Smith, J. F. (1974). Hydrocephaly. In Pediatric Neuropathology, Chapter 10. McGraw-Hill, New York.

Stahlman, M. (1966). Perinatal circulation. Pediatric Clinics of North America, 13, 753.

Stewart, A. (1972). The risk of handicap due to birth defect in infants of very low birthweight. Developmental Medicine and Child Neurology, 14, 585.

Wolf, A., and Cowen, D. (1954). The cerebral atrophies and encephalomalacias of infancy and childhood. In Neurology and Psychiatry in Childhood. Research Publications, Association for
Research in Nervous and Mental Disease, Vol. 34, p. 199. Ed. by R. McIntosh and C. C. Hare. Williams and Wilkins, Baltimore. 
Yakovlev, P. I., and Lecours, A. R. (1967). The myelogenetic cycles of regional maturation of the brain. In Regional Development of the Brain in Early Life, p. 3. Ed. by A. Minkowski. Blackwell, Oxford.
Correspondence to Professor J. F. Smith, Department of Morbid Anatomy, University College Hospital Medical School, University Street, London WC1E 6JJ.

The following articles will appear in future issues of this journal:

Factors influencing development of secondary ossification centres in fetus and newborn: a postmortem radiological study. J. Pryse-Davies, J. H. Smitham, and K. A. Napier.

Wilms's tumour -an improved prognosis : report of 22 consecutive children seen from 1967-1971. J. Martin and P. P. Rickham.

Intracellular bactericidal effects of rifampicin in both normal and chronic granulomatous disease polymorphs. G. Ezer and J. F. Soothill.

Hyperammonaemia in 20 families : biochemical and genetical survey, including investigations in 3 new families. T. Palmer, V. G. Oberholzer, E. A. Burgess, L. J. Butler, and B. Levin.

A vasopressin analogue in treatment of diabetes insipidus. $R$. Kauli and $Z$. Laron.

Rheumatoid rosette in juvenile rheumatoid arthritis. A. M. Prieur, J. F. Bach, C. Griscelli, C. Judet, J. J. Ballet, C. Bach, and P. Mozziconacci.

Difetarsone in outpatient treatment of Trichuris trichiura infestation. P. M. Leary, C. Jones, F. Douglas, and S. T. Boyd.

Urine and stool collection for metabolic studies in the newborn. M. J. Tarlow. 